\title{
Incidence of arthro-osteitis in patients with pustulosis palmaris et plantaris
}

\author{
H. SONOZAKI, ${ }^{1} \mathrm{M}$. KAWASHIMA, ${ }^{2} \mathrm{O}$. HONGO, ${ }^{1} \mathrm{H}$. YAOITA, ${ }^{3}$ \\ M. IKENO, ${ }^{4} \mathrm{M}$. MATSUURA, ${ }^{4} \mathrm{~K}$. OKAI, ${ }^{1}$ AND A. AZUMA ${ }^{1}$
}

From the ${ }^{1}$ Departments of Orthopaedic Surgery and Dermatology, Tokyo Metropolitan Komagome Hospital, Bunkyo-ku, Tokyo; the ${ }^{2}$ Department of Dermatology, University of Tokyo, Bunkyo-ku, Tokyo; the ${ }^{3}$ University of Tsukuba, Ibaraki; and the ${ }^{4}$ Departments of Dermatology and Rheumotology, Tokyo Metropolitan Otsuka Hospital, Toshima-ku, Tokyo

SUMMARY Arthro-osteitis at the anterior chest wall was found in $12(9 \cdot 4 \%)$ out of 128 consecutive patients with pustulosis palmaris et plantaris. This finding indicates that the concomitance of arthro-osteitis with PPP is not incidental but is based on some common aetiological factor. We propose a term 'pustulotic arthro-osteitis' for this condition.

Intersterno-costoclavicular ossification is a newly described rheumatic condition which shows unique ossifications between the clavicle and the first rib. From investigations of this condition we have noticed a frequent association with pustulosis palmaris et plantaris (PPP), and suggested the hypothesis that intersterno-costoclavicular ossification might be a musculoskeletal manifestation of PPP. ${ }^{1}$ This finding has prompted us to search for incidences of arthro-osteitic symptoms in patients with PPP.

\section{Materials and methods}

\section{PATIENTS}

From January to December 1979 a total of 128 patients were diagnosed as PPP in our 4 dermatology clinics at Tokyo Metropolitan Komagome Hospital, the University Hospital of Tokyo, the University Hospital of Tsukuba, and Tokyo Metropolitan Otsuka Hospital. This number was $0.58 \%$ of the total number of outpatients seen during this period.

The diagnosis was made on typical clinical features of a pustular rash including the palms and/or soles as described by Perry. ${ }^{2}$ However, unlike Caucasian patients none of our patients had concomitant lesions of psoriasis. The pustulosis of these patients was always in an acute or a subacute stage. Fortyeight were men with an average age of 45 years $(20$ paedic Surgery, Tokyo Metropolitan Komagome Hospital, 3-18-22 Honkomagome, Bunkyo-ku, Tokyo 113, Japan. to 68 ), and 80 were women with an average age of 50 ( 21 to 78 ).

All the PPP patients were checked for presence or absence of tenderness and swelling at the anterior chest wall in accordance with the list shown in Fig. 1 . If any orthopaedic abnormality was suspected, the patient was referred to the orthopaedists among the authors, and further clinical investigations, including tomography of the sternum and the clavicle, were made.

\section{RADIOLOGICAL EXAMINATIONS}

A plane $x$-ray film and a lateral view of the sternum were taken on all the patients with anterior chest
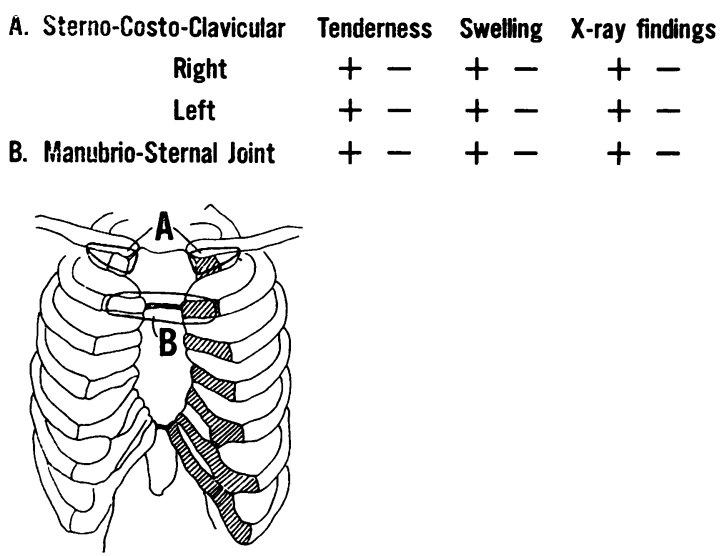

Fig. 1 Check list of arthro-osteitis at the anterior chest wall. 
wall symptoms. Lateral tomographic films of the sternum and postero-anterior tomographic films of the costoclavicular region were also taken if necessary. Apparently abnormal ossifications at the costoclavicular region (Fig. 2A) or osteomyelitis-like hypertrophy of the clavicle were considered positive $x$-ray findings. Erosive or sclerotic changes at the manubrio-sternal junction (Fig. 2B) were also regarded as positive findings.

\section{DIAGNOSTIC CRITERIA}

Patients who fulfilled either of the following 2 criteria were regarded as positive for arthro-osteitis: (1) an apparent swelling with tenderness at either side of the costoclavicular or the manubriosternal region, with or without positive $x$-ray findings; (2) tenderness without apparent swelling on the either side of the costoclavicular or the manubriosternal region, with positive $x$-ray findings at the tender site.

\section{Results}

The results are shown in Table 1 . Twelve $(9 \cdot 4 \%)$ out of the 128 patients with PPP revealed arthroosteitis symptoms. The prevalence ranged from $6.7 \%$ to $14.3 \%$, but the differences between the institutes were not statistically significant.

No significant deviation was noticed in age or sex distribution between patient groups with or without arthro-osteitis symptoms. Clinical patterns of skin eruptions were also similar between the 2 groups.

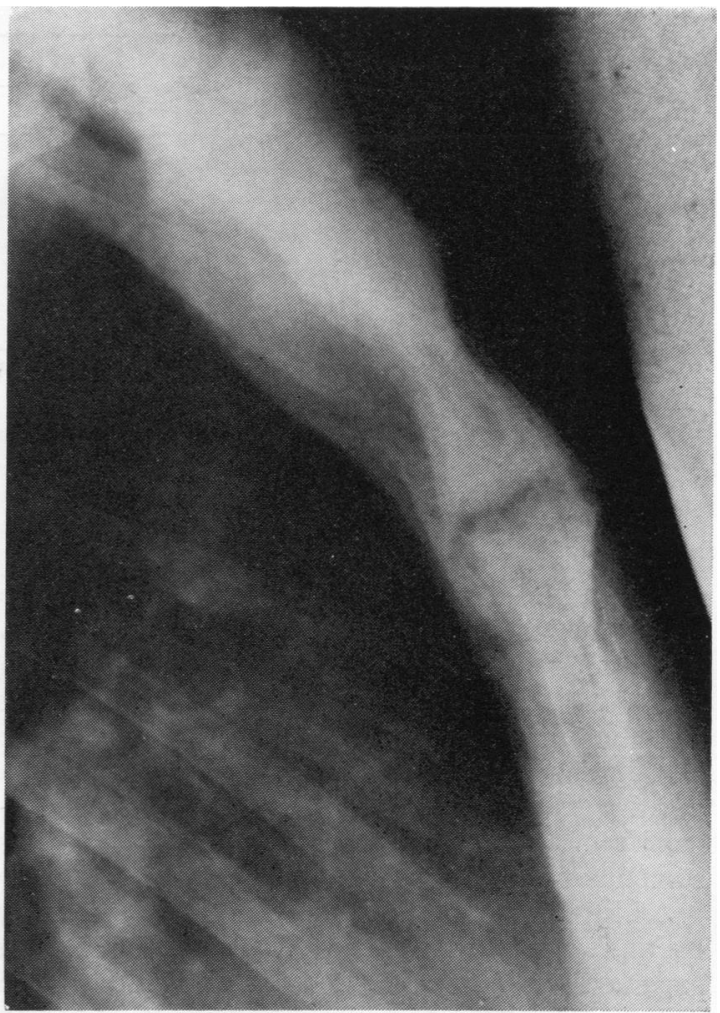

$2 B$

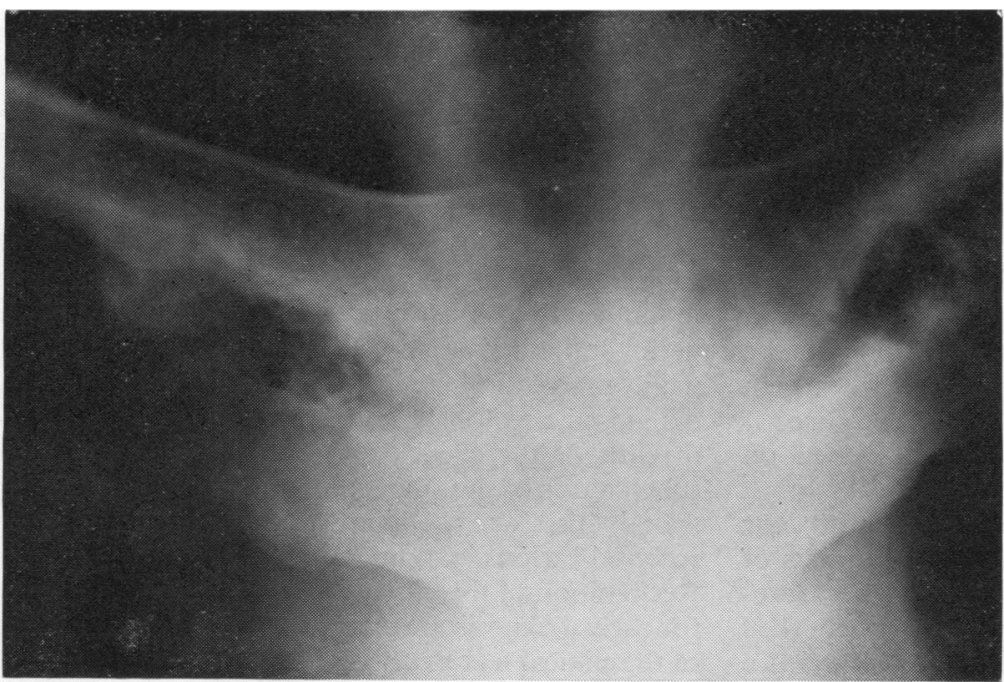

Fig. 2A. Tomography of the clavicle showing ossifications at the costo-clavicular ligament. B. Lateral view of the sternum showing sclerosis and osteophyte formations at the manubriosternal junction. 
Table 1 Incidence of pustulosis palmaris et plantaris and pustulotic arthro-osteitis

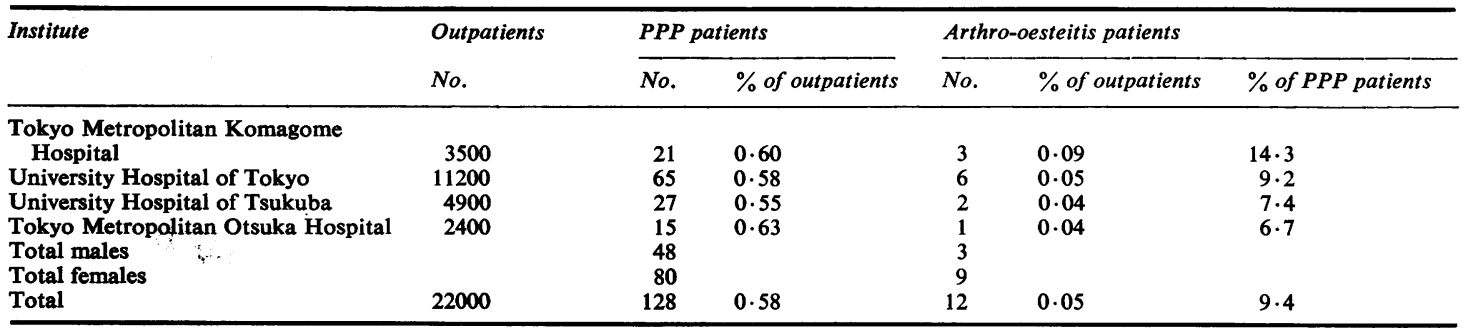

\section{Discussion}

PPP is a well known skin disease characterised by a protracted course with recurrent eruptions of sterile pustules, situated symmetrically on the palms and/ or soles of feet. Its aetiology and classification have often been discussed. ${ }^{3}$ In 1930 Barber stated this condition was a variant of pustular psoriasis. ${ }^{4}$ In 1935 Andrews and Machacek proposed the concept of an 'id' reaction. ${ }^{5}$ This suggestion was based on the fact that in some patients there was a leucocytosis, a positive reaction to bacterial toxins, and that surgical removal of focal infection such as tonsillitis sometimes improved the eruptions.

A histological similarity between PPP and pustular psoriasis has been debated. ${ }^{26} 7$ However, among Japanese dermatologists this subject has rarely given rise to discussion, and it seems generally accepted that PPP is a clinical entity apparently distinct from pustular psoriasis. ${ }^{8}$ This is probably due to the fact that psoriasis is far less frequent among the Japanese population than among Caucasians, and concomitance of PPP and psoriasis vulgaris is very rare. ${ }^{9}$

An association of arthro-osteitis with PPP has been noticed by some Japanese orthopaedic surgeons. In 1967 Sasaki reported a case of osteomyelitis of the bilateral clavicles associated with PPP. ${ }^{10} \mathrm{He}$ suggested that the osteomyelitis might be a focal infection causing the skin eruptions, supporting the 'id' theory of Andrews. In 1968, Kato et al. described another similar case, but no causative microorganism was detected in their case. ${ }^{11}$ In 1974 we described 4 cases with abnormal ossification between the clavicle and first rib. ${ }^{12}$ From the investigation of this disease we have noticed a high association of PPP. ${ }^{1}$ which stimulated us to see if this association was only incidental or based on some common aetiological factor.

All of the patients with PPP encountered in our clinics in 1979 were examined. Initially all joints of the body, including the spinal column, the sacroiliac joints, and the peripheral joints, were checked carefully. But later we have learned by experience that examining only the anterior chest wall was enough for screening the patients with arthroosteitis symptoms. As shown in the results, as many as 12 patients $(9.4 \%)$ out of 128 had anterior-chest wall symptoms. Among the 4 institutes no significant differences were observed in the frequency. The number of $9.4 \%$ is higher than the occurrence rate of psoriatic arthritis among all patients with psoriasis, which is said to be not more than 5 to $7 \% \%^{13}$ This finding indicates that association of arthroosteitis with PPP is not mere coincidence. We would like to propose a term 'pustulotic arthro-osteitis' for this condition.

In 1971 Enfors and Molin reported a 10-year follow-up cases of PPP. ${ }^{14}$ They found $13 \%$ of the patients to have complaints resembling rheumatoid arthritis. Unfortunately they did not describe details of the articular complaints. In 1977 Ishibashi et al. reported that $14(10.6 \%)$ out of 132 patients with PPP showed anterior chest wall symptoms such as manubriosternal or sternoclavicular arthritis. ${ }^{15}$ This ratio corresponds well with our result. Bergdahl et al. found 3 cases with manubriosternal arthritis among 52 patients with PPP. ${ }^{16}$

The prevalence of pustulotic arthro-osteitis is uncertain. However, we have the impression that among Japanese it might be not less frequent than psoriatic arthritis or ankylosing spondylitis, because, as the results have shown, pustulotic arthro-osteitis patients constitute about $0.05 \%$ of the total number of outpatients in dermatology clinics.

Involvement of pustulotic arthro-osteitis is not restricted only to the anterior chest wall but is often found in the spine, the sacroilliac joints, or the peripheral joints as well. In fact many of the patients with pustulotic arthro-osteitis have been misdiagnosed previously as having suppurative osteomyelitis of the clavicle, infectious spondylodiscitis, tuberculosis of the spine, disc herniation, ankylosing spondylitis, or rheumatoid arthritis, and have received various inappropriate therapeutic measures. Recognition of this disease is of primary importance for early diagnosis and correct treatment. The details of the clinical features of the disease will be described $l_{\text {in a later paper. }}{ }^{7}$ 
We are very grateful to Drs M. Konosu, K. Seino, K. Akagi, H. Hino, N. Nishida, and T. Baba for allowing us to study patients under their care and for their active collaborations.

This work was supported by a grant from the Bureau of

Public Health, Tokyo Metropolitan Government.

\section{References}

1 Sonozaki H, Azuma A, Okai K, et al. Clinical features of 23 cases with inter-sterno-costo-clavicular ossificationa new rheumatic syndrome. Arch Orthop Unfallchir 1979; 95: 13-22.

2 Perry H O. Pustular eruptions of the palms and soles. In: Fitzpatrick T M, Eisen A Z, Wolff K. Freedberg I M, Austen K F, eds. Dermatology in General Medicine, 2nd ed. New York: McGraw-Hill, 1979; 350-5.

3 Ashurst P J C. Relapsing pustular eruptions of the hands and feet. Br J Dermatol 1964; 76: 169-80.

4 Barber H W. Acrodermatitis continua vel perstans (dermatitis repens) and psoriasis pustulosa. $\mathrm{Br} \mathrm{J}$ Dermatol 1930; 42: 500-18.

5 Andrews G C, Machacek G F. Pustular bacterid of the hands and feet. Arch Dermatol 1935; 32: 837-47.

6 Lever W F. Histopathology of the Skin. 5th ed. Philadelphia: Lippincott, 1975; 143.

7 Pincas H, Mehregan A H. A Guide to Dermatohistopatho$\log y$, 2nd ed. New York: Appleton-Century-Crofts, 1976; 135-7.

8 Homma S. Kojima R, Miura O, Kiyotera K, eds. Basic Dermatology. Tokyo: Ishiyakushuppan, 1972; 2: 522 (in Japanese).
9 Takahashi Y. Pustulosis palmaris et plantaris, general remarks. Rinsho Derma 1974; 16: 307-12 (in Japanese).

${ }^{10}$ Sasaki T. A case with osteomyelitis of the bilateral clavicles associated with pustulosis palmaris et plantaris Rinsho seikeigeka 1967; 2: 333-7 (in Japanese).

11 Kato T, Kambara H, Hoshi E. A case with osteomyelitis of the bilateral clavicles associated with pustulosis palmaris et plantaris. Seikeigeka 1968; 19: 590-3 (in Japanese).

12 Sonozaki H, Furusawa $\mathrm{S}$, Seki $\mathrm{H}$, et al. Four cases with symmetrical ossifications between the clavicles and first ribs of both sides. Kanto J Orthop Traum (Tokyo) 1974; 5: 68-71 (in Japanese).

13 Sigler J W. In: Hollander J L, McCarty D J, eds Arthritis and Allied Conditions 8th ed. Philadelphia: Lee and Febiger, 1972; 725.

14 Enfors W, Molin L. Pustulosis palmaris et plantarisa follow-up study of a ten year material. Acta Derm Venereol (Stockh) 1971; 4: 37-45.

15 Ishibashi A, Nishiyama Y, Endo M, et al. Orthopedic symptoms in pustular bacterid (pustulosis palmaris et plantaris): Tietze's syndrome and arthritis of manubriosternal joint due to focal infection. $J$ Dermatol (Tokyo) 1977; 4: 53-9.

16 Bergdahl K, Bjorksten B, Gustavson K H, et al. Pustulosis palmoplantaris and its relation to chronic recurrent multifocal osteomyelitis. Dermatologica 1979; 159: 37-45.

17 Sonozaki H, Mitsui H, Miyanaga Y, et al. Clinical features of 53 cases with pustulotic arthro-osteitis. Ann Rheum Dis 